\title{
Belgeo
}

Revue belge de géographie

\section{Evolution de l'utilisation du sol le long du littoral belge}

The evolution of land-use along the Belgian coast

Isabelle Van den Steen, Benjamin Wayens et Eléonore Wolff

\section{Q OpenEdition}

1 Journals

Édition électronique

URL : http://journals.openedition.org/belgeo/12305

DOI : 10.4000/belgeo.12305

ISSN : 2294-9135

Éditeur :

National Committee of Geography of Belgium, Société Royale Belge de Géographie

Édition imprimée

Date de publication : 30 septembre 2005

Pagination : $327-348$

ISSN : 1377-2368

\section{Référence électronique}

Isabelle Van den Steen, Benjamin Wayens et Eléonore Wolff, « Evolution de l'utilisation du sol le long du littoral belge », Belgeo [En ligne], 3 | 2005, mis en ligne le 28 octobre 2013, consulté le 05 février 2021. URL : http://journals.openedition.org/belgeo/12305; DOI : https://doi.org/10.4000/belgeo. 12305

Ce document a été généré automatiquement le 5 février 2021

Belgeo est mis à disposition selon les termes de la licence Creative Commons Attribution 4.0 International. 


\section{Evolution de l'utilisation du sol le long du littoral belge}

The evolution of land-use along the Belgian coast

Isabelle Van den Steen, Benjamin Wayens et Eléonore Wolff

Les auteurs tiennent à remercier vivement les organismes (et leurs employés) à la base de la constitution de la base de données LACOAST exploitée dans le cadre de cet article, c'est-à-dire essentiellement le Centre Commun de Recherche de la Commission Européenne, l'Institut Géographique National (belge). Que nos collègues de l'IGEAT ayant collaboré au projet LACOAST soient également remerciés.

\section{Introduction}

1 Largement analysée dans ses dimensions architecturales, paysagères, économiques et sociales (Van Hove, 1980; Berndsen et al., 1985; Balthazar, 1988) la production du territoire touristique caractéristique que constitue la côte belge mérite également d'être étudiée sous l'angle de l'utilisation du sol. A l'heure où la question de l'impact du développement touristique sur la consommation d'espace préoccupe de plus en plus les aménageurs, il semble en effet utile de faire le point sur l'utilisation du sol et son évolution le long du littoral. L'objectif premier de cette démarche est de mettre en évidence la relation entre l'accroissement de la fréquentation touristique et les modalités de la consommation d'espace par le bâti. Ces modalités doivent être examinées non seulement qualitativement, comme l'ont déjà fait de nombreux auteurs, mais aussi quantitativement. De manière complémentaire, il est aussi nécessaire de mettre en évidence les évolutions d'utilisation du sol non directement liées à la fonction touristique. Cet aspect est moins présent dans la littérature scientifique.

La littérature existante insiste sur la nécessité du raisonnement multiscalaire pour comprendre l'organisation spatiale du littoral belge. Le développement touristique de cet espace a pris place dans un contexte spécifique, national (étroitesse du front de mer, intégration dans les réseaux de communication...) et international (diffusion au 
XIX ${ }^{e}$ siècle du tourisme balnéaire et de ses formes depuis la Grande-Bretagne, clientèle allemande,...). A l'échelle de la station, un schéma d'organisation particulier assez rigoureux a été maintes fois mis en évidence (Dewailly, 1989; Barbé, 2001). A l'échelle régionale, si on considère l'ensemble de la côte, il a été montré que le développement est plus spontané et moins systématique. Les stations balnéaires émergent généralement en "tête de pont", souvent à partir de localités existantes, les infrastructures de transport ne précédant pas systématiquement le développement touristique.

Néanmoins, sur le plan géographique, c'est essentiellement à l'échelle de la station que sont réalisées la plupart des analyses. Quand l'ensemble du littoral est abordé, c'est généralement dans une définition étroite (du front de mer jusqu'à 1 ou $2 \mathrm{~km}$ à l'intérieur des terres) et par généralisation des constats effectués à l'échelle de la station. Sans contester ce type d'approche, qui permet de mettre en évidence le type morphologique "station balnéaire belge " de manière rigoureuse et pédagogique (Barbé, 2001), il nous semble nécessaire de compléter l'analyse de l'espace littoral à l'échelle régionale par une démarche allant du général au particulier et intégrant l'arrière pays de manière significative à la réflexion.

La prise en compte de l'arrière-pays et l'analyse à l'échelle régionale structureront donc les démarches qui suivent en s'appuyant sur la vision quantitative que permettent les données d'utilisation du sol. Cette entrée originale de l'étude du littoral belge est complémentaire de la vision paysagère dominante dans la littérature et se prête aisément à la comparaison. Pour s'en convaincre, il suffit d'examiner la ventilation de l'usage du sol de quelques sections du littoral européen comprises entre le nord des Pays-Bas et les Pyrénées (fig. 1). Les espaces considérés correspondent tous à des côtes à plages et intègrent l'arrière pays jusqu'à $10 \mathrm{~km}$ à l'intérieur des terres. 
Figure 1. Structure de l'utilisation du sol d'un échantillon de côtes sableuses et de leur arrière pays, jusqu'à 10 km à l'intérieur des terres.

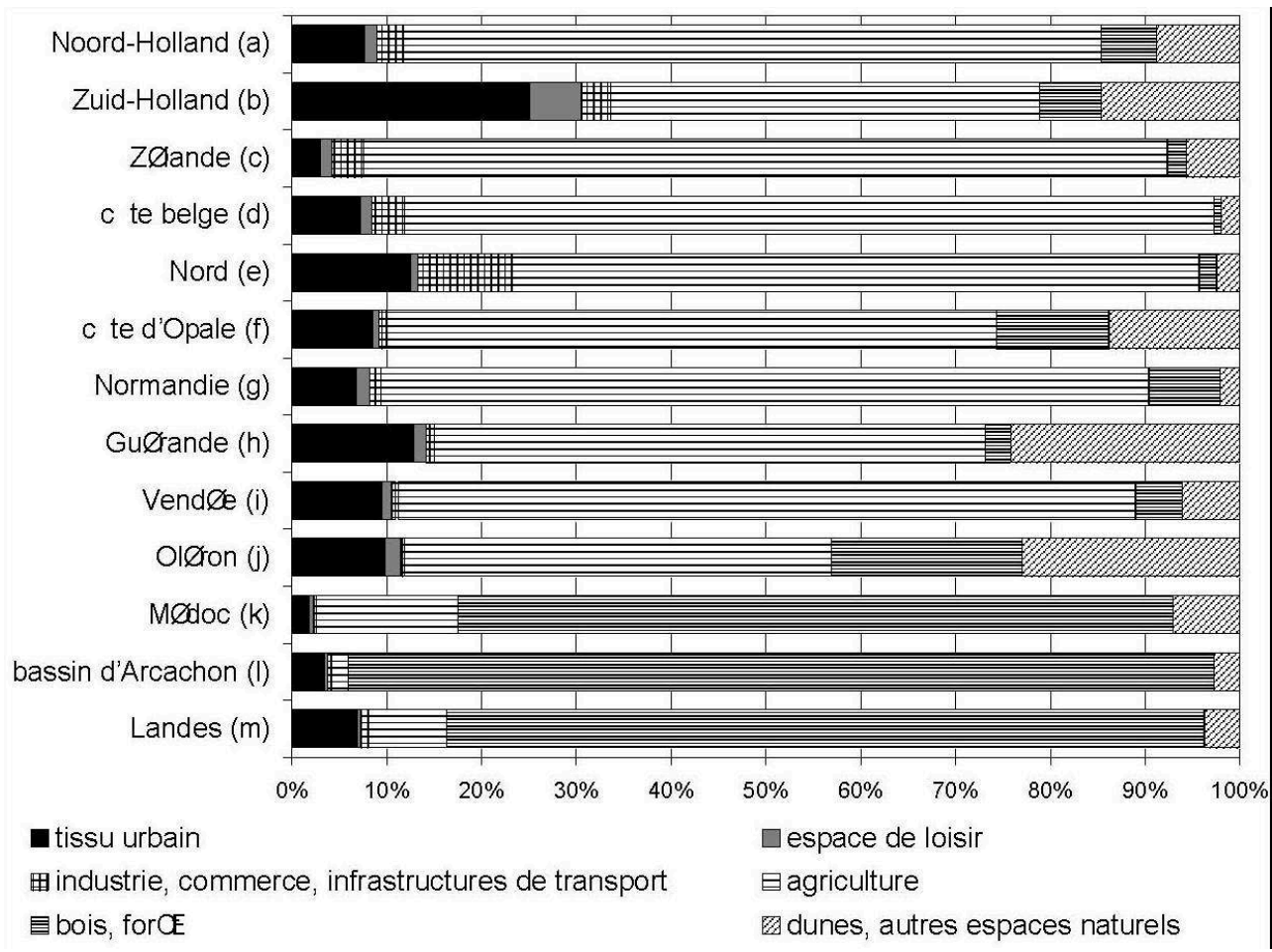

(a) de Den Helder à Wijk aan Zee, (b) de ljlmuiden à Hoek van Holland, (c) de Oostvoorne, infrastructures portuaires de Rotterdam exclues, à Cadzand, (d) de Knokke à La Panne, (e) de BrayDunes à Calais, (f) du Portel au Crotoy, (g) de Villerville à Merville, (h) de Mesquer au Pornichet, (i) de Noirmoutier à l'Aiguillon sur Mer, (j) île d'Oléron, (k) du Verdon sur Mer à le Porge-Océan, (I) bassin d'Arcachon, $(\mathrm{m})$ de Biscarosse à Boucau.

Source : EU, Join Research Centre, base de données LACOAST, pour l'utilisation du sol. Les sections de côtes ont été définies sur base de cartes topographiques et de la carte des sites naturels de France figurant p. 54 dans Knafou, 1997.

5 En ce qui concerne l'emprise des surfaces urbanisées, la zone littorale belge se situe dans la moyenne des espaces observés. L'industrie, le commerce et les infrastructures de transport y sont présents dans des proportions plus importantes qu'en moyenne, mais pas exceptionnelles pour le nord de l'Europe. La spécificité de la bande littorale belge est la forte proportion de l'espace consacré à l'agriculture et la très faible proportion d'espaces dunaires et (semi-) naturels. La confrontation de ces résultats avec les descriptions courantes $\mathrm{du}$ « second mur de l'Atlantique » belge interpelle. La spécificité du littoral belge ne réside donc pas dans l'extension de son urbanisation, du moins si l'on ne considère pas l'espace littoral de manière trop restreinte, mais plutôt dans le schéma d'organisation, caractérisé par un thalassotropisme exacerbé de l'urbanisation et une intense exploitation agricole des polders de l'arrière pays.

\section{Les données d'utilisation du sol de la côte belge}

\section{Les données d'utilisation du sol : utilité et limites}

$6 \quad$ L'approche par l'utilisation du sol permet de quantifier par une mesure commune (les surfaces) issue de différentes sources (généralement le cadastre, la télédétection ou les cartes topographiques) des usages de l'espace que les statistiques démographiques ou 
socioéconomiques décrivent de manière séparée et pas toujours aisément comparable. Cela permet d'aborder la dynamique en termes de redistribution de l'occupation physique de l'espace au cours du temps, et donc de concurrence entre les usages.

Le cadastre est une importante source de données relatives à l'utilisation du sol, caractérisée par une importante profondeur historique (Van der Haegen, 1982). Malheureusement, du fait de l'absence de publication de données agrégées à un niveau plus fin que la commune, cette source se prête peu à des exploitations à grande échelle sur de vastes zones.

8 Les données d'utilisation du sol peuvent aussi être acquises à partir de données topographiques, moyennant quelques précautions méthodologiques, de manière homogène sur une longue période historique qui correspond à l'émergence de la cartographie moderne. Même si l'indispensable traitement des documents de base est consommateur de temps et source possible d'imprécision ou d'erreurs d'interprétation, il offre l'avantage de permettre, dans une certaine mesure, de définir les objets répertoriés et leur mode de délimitation en fonction du type et de l'échelle d'analyse souhaité. Cette possibilité est évidemment plus développée pour la période postérieure à la seconde guerre mondiale, pour laquelle on dispose de couvertures photographiques régulières et exhaustives du territoire. Avant cette période, cette démarche est limitée par la précision de la légende et des modes de représentation des cartes topographiques.

9 Les données d'utilisation du sol issues de sources topographiques permettent aussi de s'affranchir des maillages administratifs dans lesquels s'inscrivent les statistiques classiques. Les fluctuations du maillage au cours du temps handicapant les démarches à long terme sont contournées et l'utilisateur peut définir une échelle d'observation plus précise, souvent infra communale, ce que ne permettent généralement pas les statistiques classiques. Enfin, ne perdons pas de vue que les cartes d'utilisation du sol sont facilement comparables aux documents de planification réglementaire tels que les cartes d'affectation (plans de secteurs, notamment).

10 Si l'approche par l'utilisation du sol présente des avantages pour des démarches de géographie historique, de suivi et de gestion de l'occupation de l'espace, il faut toutefois composer avec certaines limitations inhérentes au concept et à la manière dont les données sont collectées.

11 La première est le fait qu'il existe souvent plusieurs formes construites pour une même utilisation du sol (maisons, appartements... pour l'utilisation «bâti résidentiel », par exemple). Forme architecturale ou paysagère et utilisation du sol ne sont pas strictement associées, il s'agit de deux manières différentes et complémentaires d'observer un même espace.

Une question d'échelle intervient également dans la définition d'une utilisation du sol. On peut toujours « zoomer » et définir des usages plus détaillés tant dans l'espace que dans la légende. L'approche par l'utilisation du sol doit donc toujours s'entendre à une échelle et avec un degré de généralisation sémantique donné, ces deux aspects étant souvent dépendants (Pinchemel, 1988, p. 149 et 150).

13 Un autre inconvénient relatif aux analyses s'appuyant sur l'utilisation du sol établie sur base de données topographiques est le fait qu'il s'agisse d'une "photographie » à un moment donné. Rien de bien différent par rapport aux statistiques classiques si ce n'est le fait que l'intervalle de temps séparant deux situations successives n'est 
généralement pas d'un an ou d'une décennie (comme les recensements) mais pouvait atteindre 20 ans voire beaucoup plus. Notons toutefois que l'intervalle entre deux couvertures topographiques à tendance à se réduire fortement depuis l'émergence de la télédétection aéroportée.

Pour permettre au lecteur de juger de la qualité des opportunités et des limites des données utilisées dans notre démarche, le contenu et les méthodes qui ont conduit à l'élaboration de la base de données d'utilisation du sol LACOAST pour la côte belge seront d'abord décrits. Ensuite, la structure et l'évolution de l'utilisation du sol seront analysées de manière détaillée. Bien évidemment, nous avons confronté ces évolutions aux résultats des travaux géographiques et historiques existants. Mais devant l'abondance, l'exhaustivité et la qualité des études disponibles, cet article ne développera pas les comparaisons. Nous resterons centrés sur l'approche verticale. L'ambition n'est pas ici d'écrire une nouvelle version de l'histoire de l'urbanisation balnéaire belge, mais plutôt d'en offrir une vue synthétique et complémentaire au travers de l'utilisation du sol, à laquelle il sera aisé de rattacher les connaissances existantes.

\section{Le projet LACOAST}

L'analyse a été effectuée sur base de données d'utilisation du sol provenant du projet Européen LACOAST (LAnd cover COASTal zones). Ce projet a été réalisé pour la Commission Européenne et plus particulièrement de l'unité « Agricultural Information System» de l'Institut des Applications Spatiales du Centre Commun de Recherche. Cette base de données a été établie pour de grandes portions du littoral européen (à l'exception de la Grande-Bretagne), sur une bande de $10 \mathrm{~km}$ à l'intérieur des terres, dans le but de cartographier les évolutions de l'utilisation du sol qui se sont opérées entre 1975 et 1990 (Perdigao, 2000). Pour la Belgique, la base de données LACOAST a été réalisée conjointement par l'Institut de Gestion de l'Environnement et d'Aménagement du Territoire de l'Université Libre de Bruxelles et l'Institut Géographique National belge (Jasselette et al., 1998).

\section{La zone étudiée}

La zone interprétée couvre les $67 \mathrm{~km}$ de la côte belge, sur $10 \mathrm{~km}$ à l'intérieur des terres et $4 \mathrm{~km}$ dans la mer pour prendre en considération les infrastructures portuaires. La distance de dix kilomètres a été définie au niveau européen sur base de sites test. L'examen de la distribution de différentes classes d'utilisation du sol en fonction de la distance à la côte révèle que c'est approximativement à cette distance que se stabilisent leurs proportions respectives, traduisant la fin présumé de l'effet structurant du rivage à l'échelle envisagée. En Belgique, cette distance comprend le cordon des dunes littorales et l'essentiel des polders maritimes, à l'exception de la partie amont de la paléo-vallée de l'Yser (Tavernier \& Ameryckx, 1970). Les communes littorales concernées, bordant directement la mer, sont, d'ouest en est: La Panne, Coxyde, Middelkerke, Ostende, Bredene, Blankenberge et Knokke-Heist. 


\section{Echelle et légende}

17 L'utilisation du sol, pour un ensemble de 38 classes, a été interprétée visuellement à l'échelle du 1:100 000 à partir d'images satellitaires, de photographies aériennes et de cartes topographiques (Jasselette et al., 1998). A la différence de la plupart des autres pays européens, quatre dates de référence existent : 1995, 1976, 1950 et 1930. Les zones bâties ont également été délimitées sur base de cartes topographiques anciennes datant de 1865 et 1913. La taille minimum des polygones interprétés est de 10 hectares.

La légende d'utilisation du sol a été établie au départ de la légende CORINE Land Cover. Un niveau hiérarchique supplémentaire, comportant des classes spécifiques au milieu littoral, a été introduit pour obtenir la légende LACOAST ; ces classes ont été introduites suite à une étude de faisabilité (Jasselette et al., 1998). La classe des équipements sportifs et des loisirs a, notamment, été subdivisée en terrains de golf, champs de courses, campings, zones de sport et parcs d'attraction. De même, la classe des espaces ouverts distingue les plages, les dunes littorales et les dunes continentales. La légende complète comprend ainsi 38 postes pour le littoral belge. Parmi ces 38 classes, la légende comprend aussi bien des classes d'occupation du sol que d'utilisation du sol. La différence entre ces deux termes réside dans le fait que l'occupation du sol considère essentiellement la nature des objets tandis que l'utilisation du sol s'intéresse à la fonction des surfaces et aux activités humaines (Anderson et al., 1976). Compte tenu du niveau de détail sémantique utilisé dans les analyses et du degré d'anthropisation du territoire étudié, nous avons privilégié dans cet article le vocable "utilisation du sol», même si certaines classes de la nomenclature (plages, notamment) s'apparentent plus à de l'occupation du sol.

Dans cet article, pour la facilité de l'analyse et suite aux contraintes de la publication en noir et blanc, des regroupements de classe ont été opérés dans la légende LACOAST. Ces regroupements sont mentionnés dans la dernière colonne du tableau 1. 
Tableau 1. Légende d'occupation et utilisation du sol LACOAST.

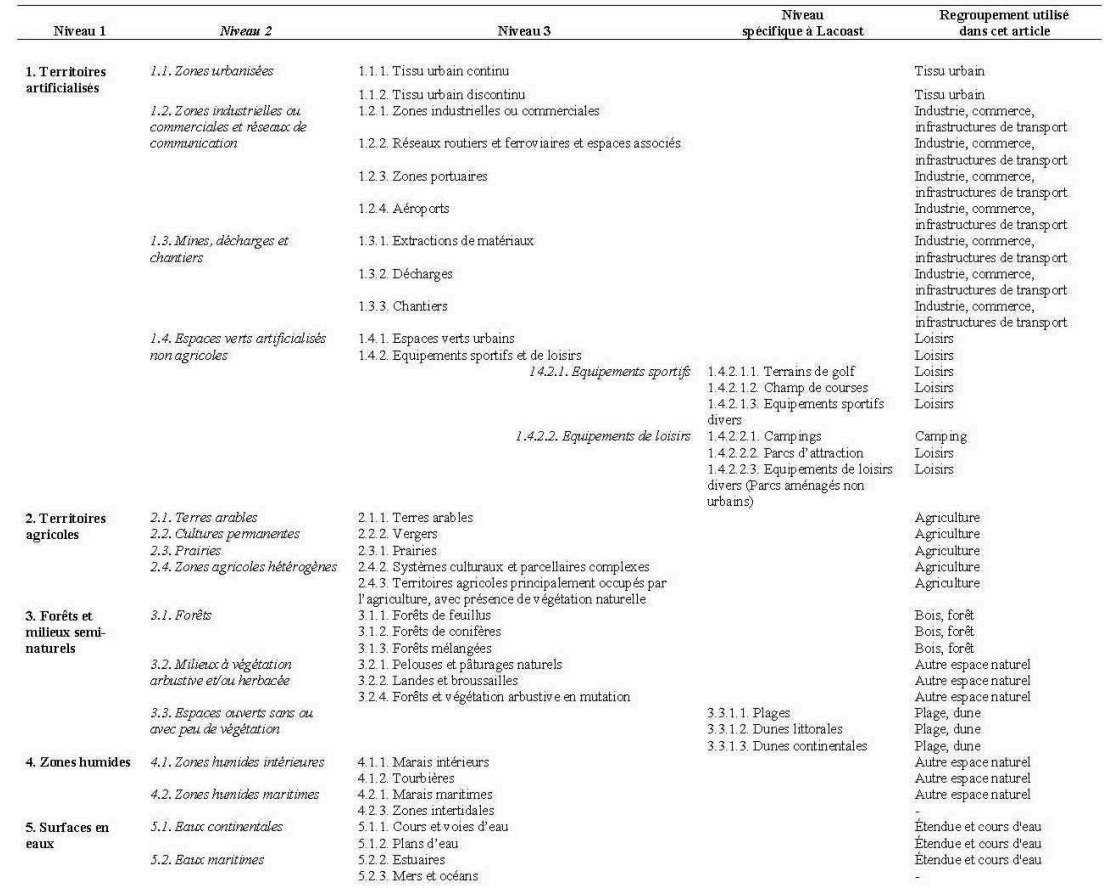

\section{Méthodes et sources}

La méthode utilisée est conforme aux recommandations unifiées au niveau européen du projet LACOAST (Jasselette et al., 1998). L'utilisation du sol est interprétée visuellement sur base d'images satellitaires, complétées par des photographies aériennes et les cartes topographiques disponibles. Pour limiter la subjectivité de l'interprétation visuelle, l'interprétation fut réalisée par les mêmes chercheurs selon une méthode d'interprétation bien définie.

La première carte d'utilisation du sol de la zone littorale est une situation 1995. Il s'agit d'une mise à jour et d'un affinement des classes spécifiques au milieu côtier par modification des polygones des données CORINE Land Cover. Cette interprétation se base sur une image satellitaire LANDSAT TM de mai 1990, sur des ortho-photographies et surtout sur la base de données topographiques au 1:50000 de l'IGN révisée entre 1993 et 1995.

22 Pour les années antérieures, la cartographie est réalisée visuellement, à partir de toutes les données disponibles, par mise à jour rétrospective ("downdating»). Chacune des situations historiques de l'utilisation du sol a été produite à partir de modifications apportées à la base de données de la période postérieure. Ainsi, seuls les changements significatifs survenus au cours de la période sont pris en compte (Fricke et al., 2002). Des interprétations indépendantes pour chaque date auraient, inévitablement, conduit à un nombre de modifications liées à la subjectivité de l'interprète et non à de réels changements d'utilisation du sol. Ainsi, en modifiant la base de données plus récente et en remontant le temps progressivement, les artefacts liés au changement de sources de données sont minimisés.

La mise à jour rétrospective de 1976 s'est basée sur la composition colorée d'une image satellitaire LANDSAT MSS de juin 1976, complétée de photographies aériennes 
(généralement au $1: 21$ 000, datant de 1974 à 1982) et de cartes topographiques au 1 : 25000 (1975-1986). Pour 1950, la mise à jour se base sur des photographies aériennes (1948-1952) et est complétée par des cartes topographiques (1950-1966). Enfin, l'utilisation du sol en 1930 a été reconstituée à partir des cartes topographiques anciennes (1925-1937) au $1: 20000$.

Cette interprétation aboutit aux quatre cartes d'utilisation du sol le long de la côte belge vers 1930, 1950, 1976 et 1995. L'utilisation de sources de données hétérogènes (images satellitaires, photographies aériennes et cartes topographiques) a nécessité une grande prudence lors de l'interprétation car les échelles, la nature des documents et, le cas échéant, les légendes diffèrent. Au niveau de la légende, les cartes topographiques anciennes ne présentent pas le même niveau de détail que les cartes actuelles. Par exemple, une confusion entre terres arables et zones stériles est possible car ces deux classes ne sont pas distinguées sur les cartes anciennes; néanmoins, le contexte général permet de lever l'ambiguïté. Autre exemple, pour distinguer le tissu urbain continu du tissu urbain discontinu sur les cartes topographiques anciennes; le tissu urbain continu a été assimilé aux zones bâties grisées et le tissu urbain discontinu a été obtenu à partir d'une généralisation des bâtiments ordinaires isolés pour autant qu'ils soient situés à moins de $200 \mathrm{~m}$ l'un de l'autre et que la zone ait une surface supérieure à 10 hectares.

Outre les difficultés d'interprétation, se pose un problème de simultanéité de la couverture spatiale. Le tableau 2 détaille les dates d'acquisition ou de publication des données. L'apparente hétérogénéité temporelle des sources doit être tempérée par le fait qu'à partir de 1950, la source de base est, pour l'essentiel, constituée par des couvertures photographiques s'étalant sur des périodes relativement peu étendues. Les autres sources mentionnées pour ces situations de référence ne sont utilisées qu'à titre complémentaire et pour lever des ambiguïtés figurant sur la source de base. On peut donc considérer que les 4 dates font généralement référence à une période précise en ce qui concerne la source de base tandis que les sources complémentaires s'étendent parfois sur plusieurs années centrées sur la date de référence. Par contre, les cartes topographiques utilisées pour déterminer la situation de 1930 s'étendent sur une période plus longue. Aussi, lorsque l'année 1930 sera évoquée, il faut garder à l'esprit qu'elle fait en réalité référence à une période s'étalant entre 1925 et 1937. Il en va de même pour l'extension du bâti pour les situations vers 1865 et vers 1913.

Tableau 2. Inventaire des données utilisées.

\begin{tabular}{|c|c|c|c|c|c|c|c|}
\hline \multirow{2}{*}{$\begin{array}{l}\text { Source de base } \\
\text { Sources }\end{array}$} & \multirow{2}{*}{$\begin{array}{c}\text { Cartes topo de } \\
1930 \\
\text { Cartes } 1930\end{array}$} & \multicolumn{2}{|c|}{$\begin{array}{c}\text { Photographies aériennes de } \\
1950\end{array}$} & \multicolumn{2}{|c|}{$\begin{array}{c}\text { LANDSAT MSS de juin } \\
1976\end{array}$} & \multicolumn{2}{|c|}{$\begin{array}{l}\text { LANDSAT TM de mai } \\
1990+\text { carte topo } 1995\end{array}$} \\
\hline & & Cartes 1950 & $\begin{array}{c}\text { Photos } \\
1950\end{array}$ & Cartes 1976 & $\begin{array}{l}\text { Photos } \\
1976\end{array}$ & Cartes 1990 & $\begin{array}{c}\text { Photos } \\
1990\end{array}$ \\
\hline Feuille IGN 4 & 1937 & $1950-54$ & $1949-57$ & $1981-84$ & 1980 & $1993-95$ & 1991 \\
\hline Feuille IGN 5 & 1937 & $1950-61$ & 1949 & $1981-85$ & 1980 & $1993-95$ & 1991 \\
\hline Feuille IGN 11 & 1933 & $1950-63$ & 1948 & $1982-85$ & 1982 & & 1988 \\
\hline Feuille IGN 12 & $1925-36$ & $1950-63$ & $1949-51$ & $1982-86$ & 1982 & $1993-96$ & $1988-91$ \\
\hline Feuille IGN 13 & 1926-34 & 1967 & 1952 & $1980-84$ & 1979 & $1993-95$ & $1988-91$ \\
\hline Feuille IGN 19 & $1925-33$ & $1965-66$ & 1952 & $1975-78$ & 1974 & & 1988 \\
\hline Feuille IGN 20 & 1925 & 1966 & 1952 & $1979-83$ & 1979 & & 1988 \\
\hline
\end{tabular}

En ce qui concerne les changements d'échelle des sources de données, la principale difficulté lors de l'interprétation fut de conserver le même niveau de généralisation des contours et des classes mixtes. Pour faire face à ce problème, l'échelle d'affichage a été fixée au 1:100000 tout au long de l'interprétation, sauf pour la situation 1930. 
L'utilisation de données complémentaires au 1:50 000 numérique pour la situation 1995 est aussi une manière de rechercher une cohérence d'échelle. Le contenu de cette base de données s'approche finalement plus de celui des cartes topographiques analogiques au $1: 25000$. L'utilisation de la nouvelle carte numérique au $1: 10000 / 1: 20000$, qui n'était pas disponible pour la totalité de la côte à l'époque de la constitution de la base de données LACOAST, aurait posé problème du fait de son contenu beaucoup plus détaillé.

\section{Pertinence du découpage temporel des données}

L'espace côtier belge étant largement le produit du tourisme balnéaire, on peut légitimement s'interroger sur la pertinence des séquences temporelle de LACOASTBelgique (étroitement dépendantes des données disponibles) par rapport aux différentes phases du tourisme balnéaire belge.

Les débuts du tourisme balnéaire belge remontant à la seconde moitié du XIX ${ }^{\mathrm{e}}$ siècle, il s'est avéré nécessaire d'accroître la profondeur historique de la base de données d'utilisation du sol LACOAST. En complément du projet LACOAST, l'extension du bâti discontinu a été digitalisée à partir des cartes topographiques au $1: 40000$ du Dépôt de la Guerre datant approximativement de 1865 (1861 à 1869 selon les feuilles) et de l'Institut Géographique Militaire datant environ de 1913 (relevés de terrain de 1911).

Outre la question de la profondeur temporelle, il convient de savoir si les situations d'utilisation du sol ainsi créées correspondent aux grandes phases du développement du tourisme balnéaire belge mise en évidence, notamment par Van Hove (1980). Cet auteur identifie 5 périodes. Nous les avons mises en perspective avec les données d'utilisation du sol disponibles.

Les trois premières périodes de développement peuvent être regroupées si l'on tient compte du type de classes sociales qui fréquente la côte : c'est-à-dire essentiellement la noblesse et la bourgeoisie, ainsi que du type de moyen de transport utilisé, le train. Par contre, il faudrait scinder les deux premières périodes de la troisième si l'on prend en considération la localisation de ces développements: les deux premières périodes voient se développer essentiellement la partie centre-est de la côte tandis que la troisième période voit se développer la partie ouest. Les données de 1865 et 1913 conviennent pour cerner l'évolution de l'espace bâti de ces deux périodes. Les années 1930 correspondent à un changement de fréquentation touristique; avec l'apparition des premiers congés payés en 1936, les classes populaires fréquentent certaines stations balnéaires. La situation dont nous disposons avec LACOAST se situe donc à l'aube de ces importants changements sociaux. Les années 1950 et 1976 correspondent à l'émergence et au développement du tourisme de masse après la deuxième guerre mondiale. Les deux dates d'analyse semblent pertinentes vu l'ampleur du développement touristique durant cette période. Les données de 1995 permettent d'appréhender la situation récente. 
Tableau 3. Concordance temporelle entre les phases du développement du tourisme balnéaire belge mise en évidence par Van Hove (1980) et les données d'utilisation du sol selon leur source.

\begin{tabular}{lll}
\hline Phases du tourisme balnéaire belge & Utilisation du sol & Source \\
\hline & & \\
$1700-1875:$ un tourisme avant la lettre & Circa 1865 & I. Van den Steen \\
$1875-1900:$ les balbutiements du tourisme & / Circa 1913 & I. Van den Steen \\
$1900-1914:$ extension du tourisme à l'ouest de la côte & Circa 1930 & LACOAST \\
$1914-1940:$ l'entre-deux-guerres & 1950 et 1976 & LACOAST \\
$1940-1980:$ l'après guerre & 1995 & LACOAST \\
& &
\end{tabular}

\section{Structure et évolution de l'utilisation du sol à la côte belge}

\section{La structuration actuelle de la bande littorale}

Aujourd'hui, les terres agricoles, le tissu urbain et les infrastructures de transport, industrielles ou commerciales occupent l'essentiel de la bande littorale de $10 \mathrm{~km}$ (fig. 2 \& 3).

Figure 2. Carte de l'utilisation du sol le long de la côte belge en 1995.

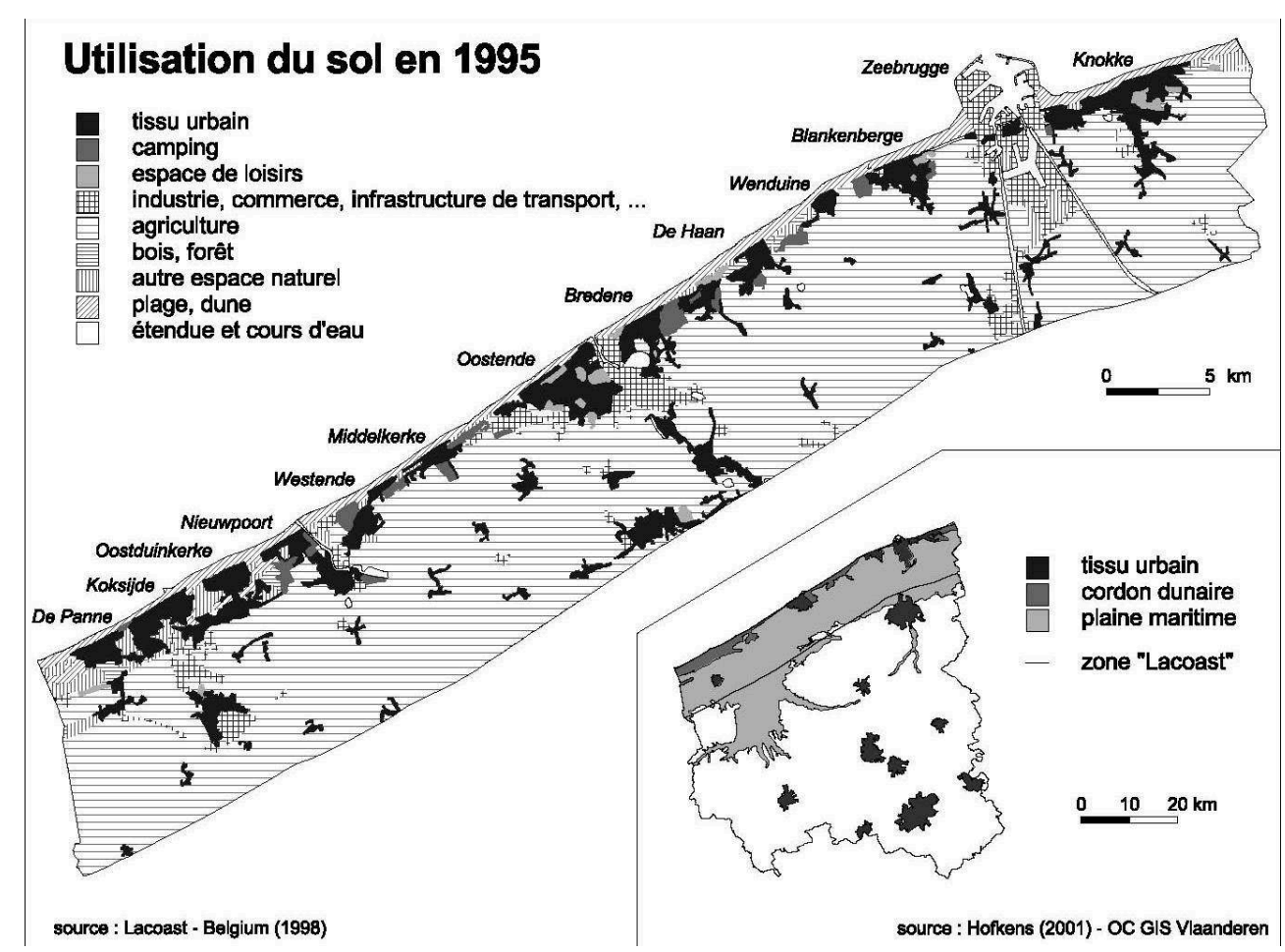

En cartouche, extension du cordon dunaire et de la plaine maritime.

Source : EU, Join Research Centre, base de données LACOAST, partie belge réalisée par l'IGN \& I'IGEAT pour la carte principale et Hofkens \& al. (2001) pour le cartouche.

Figure 3. Structure de l'utilisation du sol le long de la côte belge en 1930, 1950, 1976 \& 1995. 


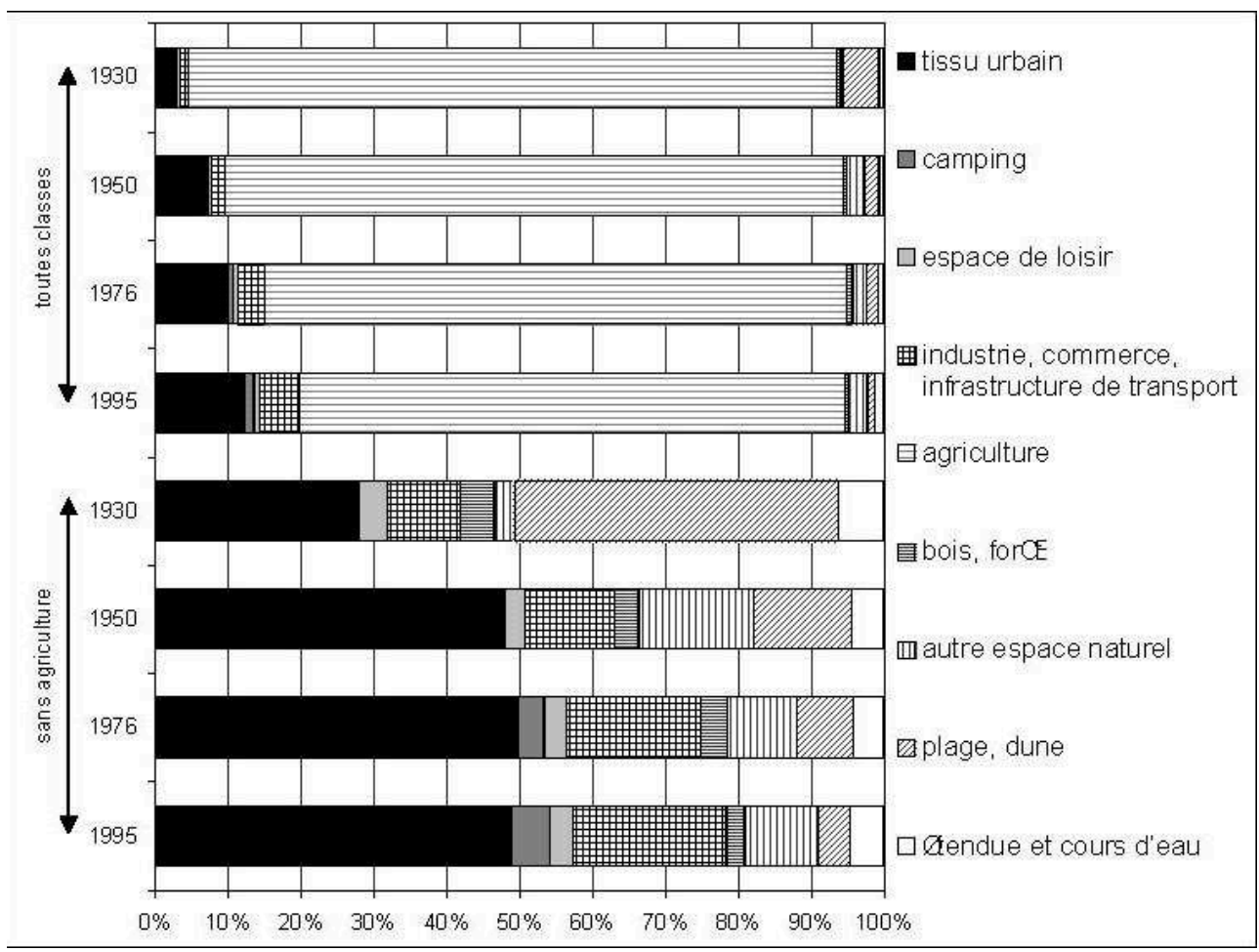

La partie supérieure du graphique est relative à l'ensemble de la zone étudiée (=100\%), la partie inférieure uniquement aux espaces non-agricoles (=100\%).

Source: EU, Join Research Centre, base de données LACOAST, partie belge réalisée par I'IGN \& I'IGEAT.

Cette image doit néanmoins être fortement nuancée. La distance au rivage structure très nettement la présence relative des différentes catégories d'utilisation du sol (fig. 4) et un gradient s'organise en bandes successives. A moins d'un kilomètre, la part du tissu urbain et des fonctions associées (campings, infrastructures de loisirs) est dominante, le reste de l'espace étant principalement occupé par les plages, les dunes et les espaces semi-naturels (souvent des dunes stabilisées ou des zones humides). Dans la bande suivante, de 1 à 3 kilomètres, l'ensemble de ces catégories régresse fortement, principalement au profit des terres agricoles. Au-delà, elles ne représentent guère plus de $10 \%$ de l'espace. Les terres agricoles se répartissent de manière complémentaire : leur présence relative augmente très rapidement avec la distance au rivage. 
Figure 4. Structure de l'utilisation du sol le long de la côte belge en 1995 en fonction de la distance au rivage.

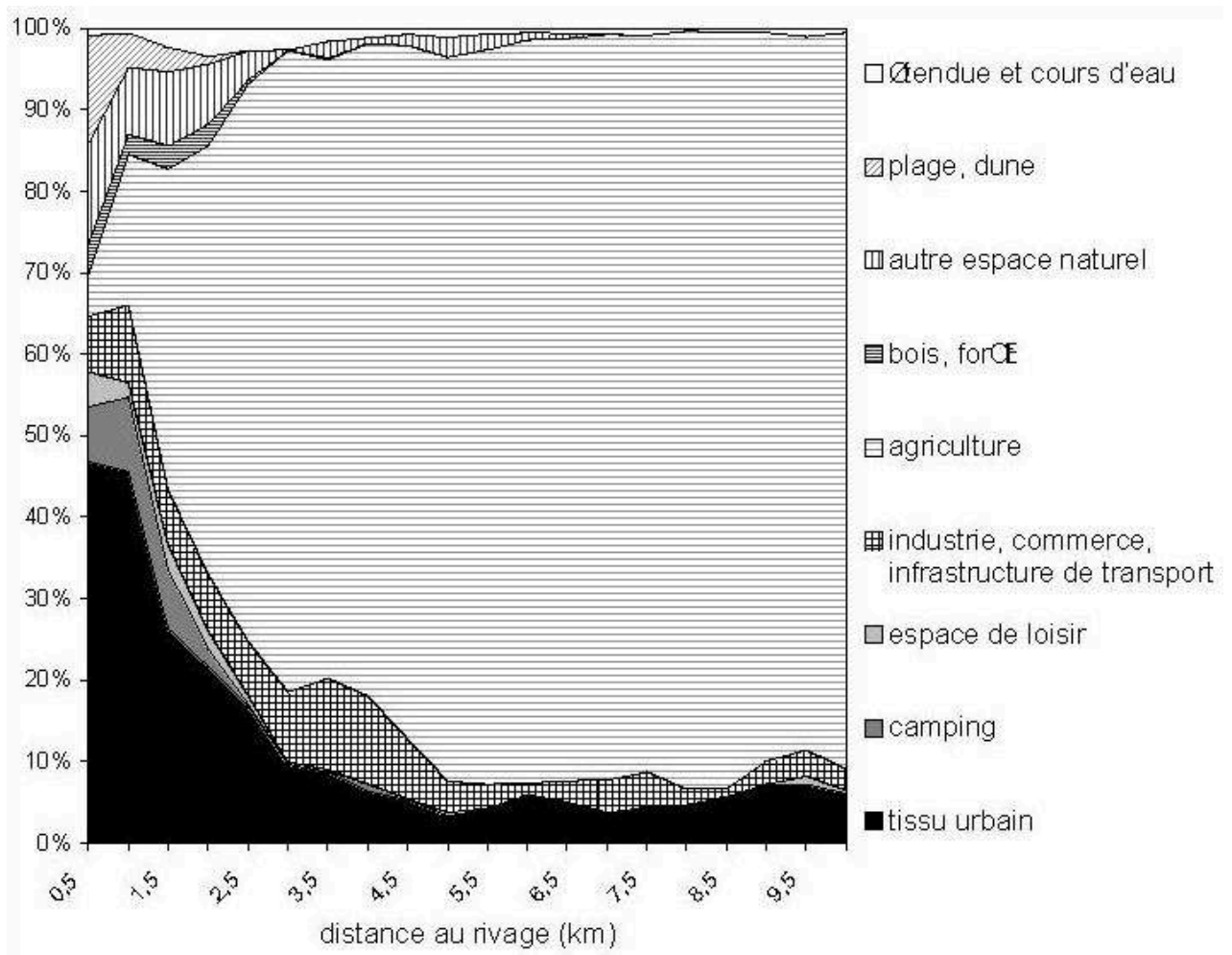

Source: EU, Join Research Centre, base de données LACOAST, partie belge réalisée par l'IGN \& I'IGEAT. le commerce vient légèrement perturber le schéma. Les infrastructures portuaires pénètrent profondément à l'intérieur des terres et atteignent leur extension maximale entre 3 et 4 kilomètres. L'arrière-port de Zeebrugge et les installations le long du canal Baudouin sont les principales infrastructures à l'origine de cette répartition spatiale, mais la contribution des ports d'Ostende et Nieuport ainsi que de l'aéroport d'Ostende (y compris les espaces commerciaux et industriels avoisinants), est significative.

La concentration du tissu urbain dans une étroite bande le long de la côte, correspondant approximativement au cordon dunaire, est évidemment symptomatique $\mathrm{du}$ thalassothropisme qui caractérise l'organisation interne de la plupart des stations balnéaires de l'Europe du Nord. Leur urbanisation se focalise le long du front de mer, souvent sur une faible profondeur: Middelkerke en est un exemple caricatural. Dans le cas de l'existence d'un pôle ancien, l'urbanisation se développe également le long de l'axe perpendiculaire le reliant au front de mer (Nieuport, Oostduinkerke...). Cette concentration urbaine s'oppose au caractère très peu urbanisé des polders où, vu la dispersion de l'habitat traditionnel, Gistel, Oudenburg et Furnes sont les seuls noyaux d'urbanisation importants. Le vide urbain de l'arrière pays est d'autant plus manifeste que Bruges, situé juste à l'extérieur de la zone cartographiée, n'a pas été à l'origine d'une périurbanisation très importante. Depuis 1830 sa population n'a pas crû de manière notable (Vandermotten \& Vandewattyne, 1985, pp. 42-46) et le maigre étalement urbain s'est essentiellement limité au sud de l'agglomération, à Sint-Andries, Sint-Michiels, Assebroeck et Sint-Kruis (Vermeersch, 2002, cartes des p. 188 à 191). 


\section{Evolution du tissu urbain et de l'utilisation du sol directement associée au tourisme}

Le tissu urbain est la classe d'utilisation du sol qui connait la plus forte croissance au cours de la période étudiée (fig. 3). Son emprise passe de 408 ha vers 1865 à 7990 ha en 1995. La plus forte croissance s'observe entre les situations 1930 et 1950 (139 ha urbanisés par an en moyenne, soit deux fois plus vite que lors des périodes ultérieures). La structuration des stations et l'occupation en chapelet du front de mer est néanmoins déjà bien visible sur base de l'extension du bâti vers 1913 (fig. 5). L'importance de cette urbanisation précoce traduit l'émergence plus ou moins rapide des stations et est à mettre en parallèle avec la mise en place des réseaux de communication, essentiellement le train (pour Ostende dès 1839, Blankenberge, Heist et La PanneAdinkerke, ensuite, essentiellement entre 1863 et 1869) et les tramways (qui desservent progressivement le reste de la côte de 1875 à 1928).

Figure 5. Evolution de l'extension du tissu urbain de 1895 à 1995.

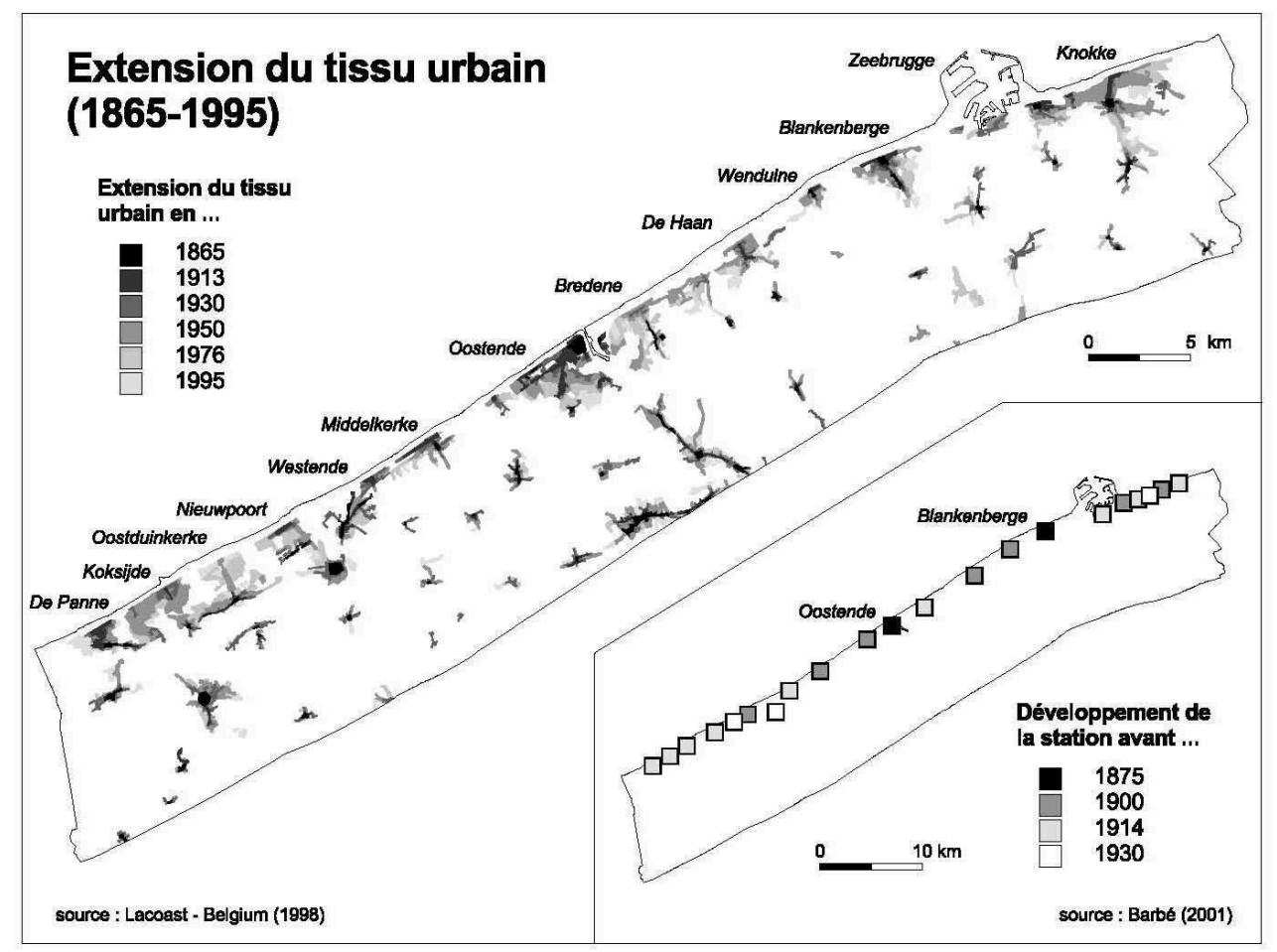

Source: EU, Join Research Centre, base de données LACOAST, partie belge réalisée par l'IGN \& I'IGEAT, pour la période 1930-1995; Van den Steen (1999) pour la période 1865 \& 1913 (carte principale) ; Barbé (2001), pour la datation développement des stations, adapté (nous avons considéré un développement significatif des stations, non pas leur stade embryonnaire).

La plupart des stations balnéaires présentent un schéma d'expansion similaire. Souvent, à partir d'un ancien noyau villageois à l'abri des dunes, les premières constructions sont établies le long de la route joignant ce noyau à la côte. Ensuite, le bord de mer connaît une extension linéaire du bâti, le long de la digue, élément caractéristique du front de mer belge. Par après, le bâti comble progressivement l'espace entre l'ancien noyau et le rivage. Durant la première moitié du $\mathrm{XX}^{\mathrm{e}}$ siècle, cette occupation rapide de l'espace se fait, à l'échelle de la station, par «blocs » successifs, 
selon des schémas urbanistiques planifiés au niveau local privilégiant l'habitat non jointif. Ce mode d'urbanisation étant très consommateur d'espace, le bâti se développe de plus en plus à l'intérieur des terres, comme l'illustre le décalage progressif au cours du temps des courbes du taux d'urbanisation en fonction de la distance au rivage (fig. 6).

Figure 6. Evolution de l'importance relative du tissu urbain en fonction de la distance au rivage, de 1895 à 1995.

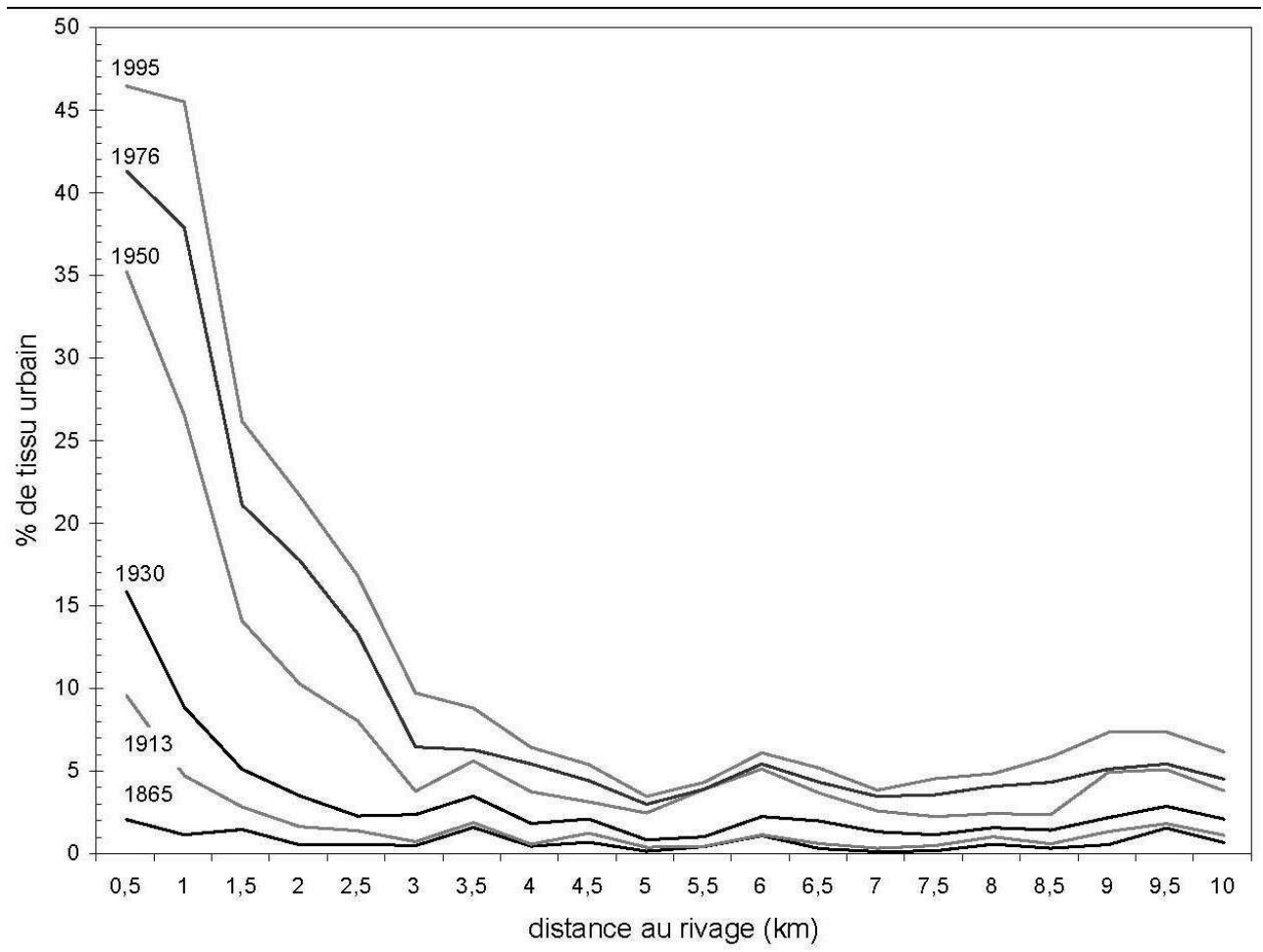

Source: EU, Join Research Centre, base de données LACOAST, partie belge réalisée par I'IGN \& I'IGEAT, pour la période 1930-1995; Van den Steen (1999) pour la période 1865 \& 1913.

Durant la seconde moitié du XXe siècle, l'urbanisation de l'espace littoral continue, mais à un rythme moins soutenu. La démocratisation du tourisme s'accompagne de la mise en place d'une urbanisation basée sur l'immeuble à appartements, bâti en hauteur en réutilisant un parcellaire fragmenté et souvent étroit. Cette régénération exceptionnelle du front de mer par des générations successives d'immeubles a été à l'origine d'atteintes paysagères maintes fois décriées mais a probablement contribué à ralentir l'expansion des surfaces urbanisées. Néanmoins, si le taux de croissance annuel moyen du bâti ralentit fortement à partir des années 50 (1,1\% par an en moyenne contre 4,2\% entre 1913 et 1950), cela représente encore plus de 70 hectares nouvellement urbanisés par an. La carte (fig. 5) montre bien le comblement progressif des interstices ainsi que quelques développements spectaculaires (front de mer d'Oostduinkerke et de Wenduine, Westhoek à La Panne).

Parallèlement à cette densification, à partir des années 70 , de nouvelles formes touristiques vont relancer l'occupation extensive de l'espace littoral: les villages de vacances. Ils contribuent pour une bonne part à l'accroissement de l'emprise de la classe «campings et villages de vacances » entre 1976 et 1995 (fig. 3). Il s'agit d'ensembles d'au moins quinze logements fixes, construits par un seul promoteur et 
destinés à des séjours de détente. Un trait morphologique les distinguant est leur disposition selon une structure pavillonnaire, sans manifestations extérieures de la propriété individuelle (clôtures). Il y a parfois regroupement de logements mais rarement des immeubles à appartements (Vanlaer, 1979). Les deux « Sunpark's » établis sur le littoral, l'un au Coq et l'autre à Oostduinkerke, constituent les fleurons de cette forme d'hébergement. Agencés autour d'une piscine, "l'Aquapark », ils possèdent chacun leur propre supermarché, leurs propres cafés et restaurants, un Kid's Club et des salles de sport. Ils ne se localisent pas à proximité de la mer mais généralement à plus de $2 \mathrm{~km}$ du rivage. Cette localisation est imposée par l'ampleur de l'espace nécessaire au fonctionnement de ces infrastructures, ce qui les exclut des zones les plus proches du littoral, saturées et/ou aux coûts fonciers élevés.

Enfin, les campings apportent au cours du temps une contribution de plus en plus significative à l'urbanisation de la côte. Ils sont absents de la base de données en 1950, tout simplement parce que, à cette date, en dehors de la période estivale ${ }^{1}$, il est impossible de distinguer cette occupation alors temporaire de l'espace de simples prairies. Par contre, en 1976 et 1995, leur présence est très nette, à moins de $3 \mathrm{~km} \mathrm{du}$ rivage mais souvent légèrement en retrait de celui-ci (fig. 4). Les 140 terrains de camping recensés le long du littoral se répartissent de manière très inégale : ils sont très nombreux dans quelques communes populaires (Le Coq, Bredene, Middelkerke) et presque inexistants dans d'autres (La Panne, Knokke). Au sein des stations, ils se situent en général à proximité des dunes du côté des polders, ce qui se marque bien à Bredene, Coxyde et Blankenberge. Parfois ils sont isolés dans les polders comme à Nieuport et Le Coq. Les campings se retrouvent quelquefois carrément dans les dunes comme à La Panne ou Middelkerke. Leur pérennisation se fait au travers des infrastructures (chemins, sanitaires...), mais surtout du formidable accroissement de la présence des caravanes résidentielles parquées à l'année. Les caravanes résidentielles constituent bien souvent des résidences secondaires à moindre coût, quand elles ne font pas office de résidences principales (Meert, 2001).

L'emprise des infrastructures de loisirs (espace vert urbain, terrain de sport, golf, hippodrome, parc d'attraction...) est également concentrée dans une étroite bande littorale où ces équipements sont intimement associés au tourisme. Toutefois, contrairement aux hébergements, cette utilisation du sol ne connaît pas d'évolution spectaculaire entre 1930 et 1997. Cela s'explique par le fait que les plus vastes de ces infrastructures étaient déjà en place au début du XXe siècle. Le Royal Zoute Golf Club est inauguré en 1899, l'hippodrome d'Ostende en 1883, le Royal Ostende Golf Club en 1903. Contribuant avec ces deux derniers à l'aménagement d'Ostende, le parc MarieHenriette est bien évidemment associé à l'action de Léopold II et donc déjà présent à la fin du XIX siècle.

\section{Evolution des infrastructures de transport et des zones d'activités industrielles et commerciales}

L'évolution observée la plus importante, hors tissu urbain, est celle des infrastructures de transport et des zones d'activité industrielle et commerciale.

Les zones portuaires occupent des superficies importantes. L'extension la plus marquée est celle de Zeebrugge, qui s'étend constamment depuis sa création en 1907. La volonté à la base de la construction du port de Zeebrugge remonte à l'indépendance de la 
Belgique et est relativement indépendante de la logique touristique qui structure l'aménagement du reste de la côte. Sa construction était essentiellement guidée par un souci d'indépendance vis-à-vis des Pays-Bas, qui contrôlent l'Escaut, en permettant l'arrivée de bateaux à gros tonnage et à grande vitesse (Charlier, 1987). Le port de Zeebrugge a connu une extraordinaire extension au cours des 30 dernières années. En 1977 la construction d'une nouvelle écluse maritime a été entamée, dans le but de permettre l'accueil de grands navires, en particulier les pétroliers. Deux nouveaux môles sont construits, s'avançant de plus de $2 \mathrm{~km}$ dans la mer, protégeant un port de haute mer. Une multitude d'espaces spécialisés sont construits ou étendus (terminal conteneur, gazier, roll on-roll off). A côté des énormes superficies concernées par le développement de Zeebrugge, les autres développements portuaires sont plus modestes et se limitent essentiellement à Ostende. A Nieuport par contre, l'essentiel du développement portuaire récent se fait dans un cadre touristique (extension du port de plaisance entre 1976 et 1995).

43 Les zones industrielles occupent de grandes superficies à proximité d'Ostende, de Zeebrugge et de Furnes et croissent régulièrement surtout à partir de 1950. A ces zones industrielles souvent associées aux infrastructures portuaires sont venues s'ajouter, depuis la fin des années 70, plusieurs zones commerciales périphériques (dont notamment le parc commercial de Middelkerke).

Les aéroports occupent également un espace important dans la bande littorale de $10 \mathrm{~km}$ étudiée. Ils connaissent deux phases d'extension. En 1950, la superficie occupée par les aéroports est à son maximum (199 ha), pour retomber en 1976 à un minimum de 18 ha et remonter à 91 ha en 1995. Outre l'aérodrome militaire de Coxyde ${ }^{2}$, construit au cours de la seconde guerre mondiale, Knokke et Ostende sont les deux localités concernées par ce type d'infrastructure.

En 1930 existait à Knokke un aérodrome de superficie considérable. Cet aérodrome, qui a vu le jour en 1929, avait une vocation essentiellement touristique, en tant que moyen d'accès au littoral pour les classes aisées. Il est actuellement remplacé par des prairies, des forêts de conifères et des équipements sportifs (karting) mais aussi par du bâti discontinu. L'agrandissement observé entre 1930 et 1950 est lié à l'activité militaire pendant la guerre. Aménagé par la Compagnie du Zoute, il fut loué à l'Etat pour une durée de 30 ans. En 1960, lorsque le contrat avec l'Etat belge prit fin, le terrain fut fermé car il freinait le développement de Knokke vers l'est. Contrairement à d'autres stations huppées (Le Touquet ou Deauville), Knokke n'a pas conservé son aéro-club.

L'aéroport d'Ostende apparaît sur les cartes d'utilisation du sol entre 1930 à 1950. C'est en 1921-22 qu'Ostende eut son premier aérodrome : une grande prairie sur l'ancienne commune de Stene. Il s'est étendu par la suite. Dès 1927, la Sabena, créée quatre années plus tôt, assure la liaison Bruxelles-Londres avec escale à Ostende. Le terrain de Stene devint vite trop petit, un nouveau terrain fut trouvé à Middelkerke. Les travaux d'arpentage commencèrent en 1939 et durant la guerre les Allemands réalisèrent le transfert à Middelkerke. En 1976, son extension apparaît en chantier et en 1990 l'aéroport d'Ostende occupe l'étendue qu'on lui connaît aujourd'hui. 


\section{Evolution des espaces non-urbanisés}

47 C'est bien évidemment principalement au dépend des utilisations du sol que l'on peut qualifier de «non-urbanisées » que croissent celles liées à la mise en valeur touristique et industrialo-portuaire de la côte belge.

Durant la période 1930-1995, ce sont les territoires agricoles qui régressent le plus fortement : leur extension diminue en moyenne de $141 \mathrm{ha} / \mathrm{an}$.

Les dunes, plages et autres espaces naturels perdent en moyenne 16 ha par an. Il est nécessaire de regrouper ces deux catégories dans la mesure où, tant du fait d'artefacts liés à l'interprétation que de la stabilisation des dunes, certains espaces dunaires se voient caractérisés par une végétation arbustive et herbacée, et ne sont plus considérés comme dunes au sens de la nomenclature LACOAST (zones de sables non couvertes par la végétation). Il convient de noter que ces moyennes varient fortement au cours de la période étudiée. La plus forte diminution de l'emprise des dunes et autres espaces naturels s'observe entre 1930 et 1976 (-25 ha/an). Tout porte à croire, compte tenu de l'évolution du bâti et de sa localisation à la fin $\mathrm{XIX}^{\mathrm{e}}$ et au début $\mathrm{XX}^{\mathrm{e}}$ siècle, que c'est durant la première moitié $\mathrm{du} \mathrm{XX}^{\mathrm{e}}$ siècle que les dunes ont payé le plus fort tribut à l'urbanisation balnéaire. Le rythme de disparition de ces espaces diminue ensuite moins fortement $(-4,8 \mathrm{ha} / \mathrm{an})$. Ce ralentissement n'est pas entièrement dû à un épuisement du «stock » de dunes et d'espaces naturels car le taux de croissance annuel moyen calculé en terme relatif décroît également au cours du temps : il passe de $-0,8 \%$ (1930-1950) à -0,2 \% (1976-1995). A contrario, loin de se ralentir, la disparition d'espaces agricoles augmente au cours du temps, passant de -126 ha/an entre 1930 et 1950 à -173 ha/an entre 1976 et 1995.

Replacées dans le contexte plus global de la côte, ces évolutions différentielles ne sont guères étonnantes. Une disparition accrue des terres agricoles au cours du temps va de pair avec la croissance du tissu urbain vers l'intérieur du pays et, surtout, de plus en plus, avec l'édification des grandes infrastructures de transport. Ces dernières, tout comme les espaces industriels pénètrent beaucoup plus largement à l'intérieur des terres, leur bon fonctionnement n'étant pas directement conditionné par la proximité immédiate au rivage. Une cohabitation trop proche avec les espaces à vocation touristique n'est d'ailleurs souvent pas souhaitable. Chassées de la proximité du rivage, leur caractère planifié et/ou d'intérêt public leur permet toutefois, moyennant des coûts de viabilisation parfois élevés (drainage), de se substituer sans trop de problème aux bonnes terres agricoles des polders.

51 Les dunes et espaces naturels qui persistent occupent actuellement un espace côtier interstitiel, entre les stations balnéaires qu'elles contribuent à isoler les unes des autres. Périphériques à l'échelle de la station, elles le sont aussi à l'échelle de la côte belge, le Zwin et le Westhoek constituant les sous-ensembles continus les plus importants. A proximité immédiate du rivage et offrant un modeste relief valorisable, les dunes ont évidemment très tôt cédé la place au tissu urbain, pour l'essentiel avant 1976. Même si quelques grignotages spectaculaires ont défrayé la chronique (les buildings à l'ouest de La Panne), force est de constater que l'essentiel des dunes et des espaces naturels existants à la fin des années septante ont été préservés. Ceci est largement dû au ralentissement de l'extension spatiale du bâti. Il faut aussi constater qu'il n'a pas fallu attendre la mise en place des plans de secteur fin des années 70 pour qu'un intérêt pour la préservation de ces espaces émerge. 
52 A la fin de sa vie, en 1909, Léopold II avait commandé une étude de la structure foncière de ces espaces afin d'en préparer la protection via une acquisition par l'Etat. Plusieurs études scientifiques, comme celle de J. Massart (1912) ont également dressé des listes de sites en vue de leur préservation. L'inventaire des paysages remarquables de $\mathrm{E}$. Kesteloot est publié en 1963 par le Survey National, organisme national chargé de réunir la documentation nécessaire aux démarches d'aménagement du territoire. La comparaison de cet inventaire avec une carte topographique actuelle permet de faire un rapide bilan du devenir des sites naturels considérés comme important à l'époque. Sur la quinzaine de sites majeurs répertoriés à l'époque, seul deux ont complètement disparus (remplacés par des infrastructures portuaires), même si on doit constater quelques grignotages significatifs (surtout dans le Westhoek). Notons aussi que plusieurs massifs dunaires non-repris par Kesteloot existent toujours.

53 Les plans de secteur instaurés à la fin des années 70 ne feront donc qu'assurer une pérennisation tardive des espaces naturels de la côte, démarche commencée près de 80 ans plus tôt. L'étendue des espaces naturels s'est stabilisée et dans les prochaines années, on les verra peut-être même croitre légèrement. La région flamande renforce actuellement la logique de protection (le plan de secteur et autres réglementations, comme le « Duinendecreet ») par une logique d'acquisition de sites, y compris certaines « friches » touristiques ou militaires rendues à la nature.

\section{Conclusions}

Sur base des données d'utilisation du sol aux différentes dates produites pour la côte et son arrière pays dans le cadre du programme "LACOAST », les analyses d'évolution ont permis de compléter ou de nuancer la perception géographique de l'espace littoral belge.

55 Tout d'abord, l'approche à l'échelle régionale souligne que si globalement le tissu urbain n'occupe pas un espace proportionnellement plus important dans la bande littorale de $10 \mathrm{~km}$ belge que dans d'autres portions du littoral européen, il est très fortement concentré dans les quelques centaines de mètres les plus proches du rivage alors que l'immédiat arrière pays est très peu urbanisé. Cela différencie nettement, par exemple, le littoral belge du littoral néerlandais, où l'urbanisation prend généralement place derrière le cordon dunaire, scrupuleusement préservé.

56 Si une bonne partie de l'urbanisation du littoral est en relation directe avec sa mise en valeur touristique, force est de constater que le taux d'urbanisation varie fortement au cours du temps et ne traduit pas fidèlement, loin s'en faut, l'importance de la fréquentation touristique. Si on confronte l'évolution de l'urbanisation à l'évolution de la fréquentation touristique, on constate que la consommation d'espace «touristique " est tout d'abord relativement extensive. Cette première phase, se terminant avec la seconde guerre mondiale et basée sur un modèle pavillonnaire, est particulièrement consommatrice d'espace. C'est durant cette phase que l'essentiel du cordon dunaire littoral est progressivement colonisé. Après-guerre, la massification du tourisme ne se traduit pas par une consommation d'espace proportionnelle. Le modèle, tant décrié esthétiquement (voir le titre de l'article de Doucet, 1986), de l'immeuble à appartements se généralise sur le front de mer et ses environs immédiats. La réutilisation de l'espace déjà bâti proche du littoral, dans une logique de plus en plus intensive, permet d'absorber une bonne part de la demande d'hébergements ${ }^{3}$ et limite 
l'extension horizontale des stations. Si le mode de croissance "régénératif » est largement dominant après-guerre, certaines formes de croissances extensives persistent, voire se renforcent aujourd'hui. Le développement des villages de vacances et la pérennisation des campings contribuent de manière non-négligeable à l'urbanisation de l'espace littoral, même si c'est moins spectaculaire car plus éloigné du rivage. Les espaces dunaires résiduels étant de mieux en mieux protégés, ce sont maintenant les espaces agricoles à l'interface dunes-polders qui sont les plus concernés par l'urbanisation.

Enfin, l'analyse en termes de redistribution de l'occupation physique de l'espace au cours du temps, et donc de concurrence entre les usages, que permet aisément l'examen de la structure de l'utilisation du sol, a mis en évidence d'autres évolutions significatives. L'importance du développement des infrastructures de transport, industrielles ou commerciales, dont une bonne part (Zeebrugge, aéroports...) est quasiment déconnectée de la logique touristique, doit être soulignée. Ce sont elles qui dans les années récentes ont le plus contribué à la réduction des surfaces agricoles, jusque là relativement préservées par leur éloignement du rivage.

Ces éléments supplémentaires, s'ils ne remettent bien évidemment pas en question les apports des travaux antérieurs sur le littoral belge, montrent la nécessité de plus en plus forte d'intégrer l'arrière pays dans sa compréhension et sa schématisation. L'approche relativement exhaustive permise par les données utilisées montre aussi très clairement le glissement historique des enjeux d'aménagement des espaces dunaires proches de la mer vers l'interface dune-polder (jusqu'il y a peu relativement épargné par la mise en place du modèle de développement « régénératif »), voire plus loin en ce qui concerne les grandes infrastructures.

\section{BIBLIOGRAPHIE}

ANDERSON J.R., HARDY E.E., ROACH J.T., WITMER R.E. (1976), A land use and land cover classification system for use with remote sensor data, Geological Survey (USGS), Washington DC.

BALTHAZAR H. (dir.) (1988), Histoires d'eaux, Bruxelles, CGER.

BARBÉ M. (2001), « Des espaces spécifiques : les espaces touristiques littoraux », Géo, 2001-2.

BERNDSEN J., SAAL P. \& SPANGENBERG F. (1985), Met zicht op zee, s'Gravenhage, Staatsuitgevrij, $96 \mathrm{p}$.

CHARLIER J. (1987), « Les problèmes d'aménagement suscités par la croissance du port de Zeebrugge », Norois, 34, pp. 239-251.

DOUCET B. (1986), « Zeventig kilometer vervelling. Bouwen langs Belgische kust », Ons Erfdeel, XXIX, 3, pp. 405-411.

DEWAILLY J.M. (1989), Tourisme et aménagement en Europe du Nord, Paris, Masson, 248 p.

GRIMMEAU J.P. (2003), « Tourisme et démographie à l'échelle locale en Belgique », Espace, Population, Sociétés, 2, pp. 263-275. 
FRICKE R., WOLFF E. (2002), « The MURBANDY project : development of land use and network databases for the Brussels area using remote sensing and aerial photography ", International Journal of Applied Earth Observation and Geoinformation, 4, pp. 33-50.

GOBIJN R. et al. (1988), "Stations balnéaires en Belgique du XVI ${ }^{\mathrm{e}}$ au XX $\mathrm{XX}^{\mathrm{e}}$ siècle ", in Histoires d'eaux, CGER, Bruxelles, pp. 9-70.

HAULOT A., VAN HOVE N. \& VERHEYDEN (1977), L'occupation touristique d'une bande côtière et ses conséquences écologiques, Connaissance du tourisme, Ministère de la Communauté française, Bruxelles, $31 \mathrm{p}$.

HOFKENS E. \& ROOSENS I. (ed.) (2001), Nieuwe impulsen voor de landschapzorg. De landschapsatlas, baken voor een verruimd beleid, Bruxelles, Ministerie van de Vlaamse Gemeenschap, afdeling Monumenten en Landschappen.

JASSELETTE J.C., WOLFF E., SINTZOFF M. (1998), Lacoast Belgium : rapport final, rapport inédit pour le Joint Research Centre de l'Union Européenne.

KESTELOOT E. (1963), Inventaris van de landschappen : tome IX, West Vlanderen, Bruxelles, Nationale Survey.

KNAFOU R. et al. (1997), Atlas de France : tome VII, Tourisme et Loisirs, Paris, La Documentation Française.

MASSART J. (1912), Pour la protection de la nature en Belgique, Bruxelles, Lamertin, 308 p.

MEERT H. (2001), « Space and the reproduction of residual housing outside cities. The case of inhabited campsites in Flanders », Belgeo, 3, pp. 277-293.

NORRO P. (1992), « Toerisme en natuurbehoud aan de Vlaamse Kust : een utopie? », Planologisch Nieuws, 1, pp. 41-52.

PERDIGAO V. \& CHRISTENSEN S. (2000), The Lacoast atlas : land cover changes in European coastal zones, European Commission Joint Research Centre, Ispra, $272 \mathrm{p}$.

PINCHEMEL P. \& G. (1988), La face de la terre, Paris, Armand Colin, 517 p. (édition de 1995).

TAVERNIER R. \& AMERIJK J. (1970), « Kust, duinen, polders », Atlas de Belgique, planche 17.

TOWNER J. (1996), An historical geography of recreation and tourism in the western world 1540-1940, Wiley, $312 \mathrm{p}$.

VAN DEN STEEN I. (1999), Analyse de l'évolution de l'occupation du sol depuis l'émergence du tourisme balnéaire à la côte belge, mémoire de licence en géographie, ULB, 155 p.

VAN DER HAEGEN H. (1982), « L'utilisation du sol en Belgique et son évolution depuis 1834 sur base des données cadastrales ", Bulletin de statistique, 1.

VANDERMOTTEN C., VANDEWATTYNE P. (1985), « Les étapes de la croissance et de la formation des armatures urbaines en Belgique », Bulletin du Crédit Communal, 154, pp. 41-62.

VAN HOVE N. (1973), Het belgische kusttoerisme : vandaag en morgen, Brugge.

VAN HOVE N. (1980), « Le littoral belge », Hommes et Terres du Nord, 4.

VANLAER J. (1979), « Les villages de vacances », Revue Belge de Géographie, 5, pp. 152-210.

VERMEERSCH V., RIJCKAERT M. et al. (2002), Bruges, Antwerpen, fonds Mercator, 319 p.

WOLFF E., JASSELETTE J.C. \& BAYERS, E. (1995), Corine Land Cover Belgium, Bruxelles, IGN, disponible sur : www.ngi.be/Common/articles/CA_Td/CLC90rapfinal.pdf. 


\section{NOTES}

1. Les vols à vocations topographiques, les seuls disponibles exhaustivement à cette date, privilégient souvent l'hiver et le printemps de façon à ce que l'interprétation ne soit gênée par le feuillage des arbres.

2. ...qui lui aussi contribue au fonctionnement touristique de la côte : la seule unité qu'il héberge est dévolue au secours en mer (notamment au bénéfice des plaisanciers et autres véliplanchistes en difficulté).

3. La demande en logement à la côte n'est bien évidemment pas exclusivement liée au tourisme car une partie de l'urbanisation est entretenue par le développement de la population permanente du littoral. Néanmoins, une part de cette croissance démographique est clairement associée au tourisme, au travers des migrations de retraite, identifiées depuis les années 70 et «se dirigeant préférentiellement vers des communes au cadre agréable, aussi caractérisées par la présence de résidences secondaires et d'autres formes de tourisme de séjour » (Grimmeau, 2003)

\section{RÉSUMÉS}

Utilisant la base de données LACOAST, l'article retrace l'évolution de l'utilisation du sol d'une bande de $10 \mathrm{~km}$ de profondeur correspondant à l'espace littoral belge (cordon dunaire et polders). Proportionnellement, le tissu urbain n'y occupe pas un espace plus important que dans d'autres portions du littoral nord européen, mais il se concentre fortement à proximité immédiate du rivage, l'arrière pays étant très peu urbanisé. L'emprise des différentes phases d'urbanisation est comparée et la relative parcimonie foncière du modèle d'urbanisation régénératif de la période d'après guerre mise en évidence. Enfin, le glissement récent de l'urbanisation vers les polders, longtemps préservés, et l'importance de l'emprise des infrastructures non directement liées au tourisme (Zeebrugge, notamment) sont soulignés. Ces constatations sont établies sur des bases quantitatives, en termes de superficies utilisées, et non pas selon une vision paysagère qualitative, plus communément répandue.

Using the data from the LACOAST database, the paper traces the land-use evolution of a $10 \mathrm{~km}$ broad land strip forming the Belgian coast (dune cordon and polders). Proportionately, the urban fabric is not denser here than in other parts of the coasts of northern Europe, but it is highly concentrated at the immediate proximity of the coastline, while inland urbanization is few important. We will compare the results of the successive urbanization stages, and highlight the relative parsimony of the regenerative urbanization model of the post-war period. Finally, we will look into the recent urbanization trend towards the polders area, for a long time protected, and the significance of the infrastructures non directly related to tourism (e.g. Zeebrugge). These statements are produced from quantitative bases in terms of used surfaces, and do not proceed from a more usual qualitative landscape analysis.

\section{INDEX}

Keywords : land-use, coastal region, tourism, urbanization, Belgium

Mots-clés : utilisation du sol, littoral, tourisme, urbanisation, Belgique 


\section{AUTEURS}

\section{ISABELLE VAN DEN STEEN}

Université Libre de Bruxelles, IGEAT, Système d'Information Géographique et Télédétection, boursière FRIA, ivdsteen@ulb.ac.be

\section{BENJAMIN WAYENS}

Université Libre de Bruxelles, IGEAT, Géographie Appliquée et Géomarketing, bwayens@ulb.ac.be

\section{ELÉONORE WOLFF}

Université Libre de Bruxelles, IGEAT, Système d'Information Géographique et Télédétection, ewolff@ulb.ac.be 